\title{
A Unified Framework for the Construction of Higher-order Methods for Nonlinear Equations
}

\author{
Wei $\mathrm{Li}^{*}$ and Hong Chen
}

Institute of Operational Research \& Cybernetics Hangzhou Dianzi University, Hangzhou, 310018, P.R. China

\begin{abstract}
In this paper, a unified framework is proposed for constructing higher-order modifications of Newton's method based on the existing iteration formulas. Some recently developed iterative methods are special cases of our approach. Also, many new iterative methods can be developed by using the proposed unified framework.
\end{abstract}

Keywords: Nonlinear equations, Iterative method, Newton's method, Order of convergence.

\section{INTRODUCTION}

Much attention has been given to develop iterative methods for solving nonlinear equations in these years, see [1-37] and the references therein. Ostrowski [1] introduced some third-order scheme. King [2] developed a family of fourth-order methods. Jisheng Kou [29] presented a method with the order of convergence five. Neta [3] developed oneparameter family of sixth-order methods for nonlinear functions. Popovski [7] showed some three-step methods, as well as Neta's methods, but have an asymptotic convergence rate 7 which is better than the 6 of Neta. Also, Popovski [8] presented a class of two-step, sixth order methods.

Among wide variuos of papers have been published in the recent years, we mention some progress about mutli-step methods. Abbasbandy [19] and Chun [22] have proposed and studied several one-step and two-step iterative methods with higher order convergence. Noor and Noor [26], Yun [33] and Hueso et al. [34] studied some three-step iterative methods with third order convergence.

These mutli-step methods have been suggested by combining the well-known Newton method with other methods. Motivated by the research going on in this direction, we present a unified approach for constructing new higher-order methods by using the existing iteration formulas. It provides a convenient tool to develop new iteration formulas, which improve the Newton method. Also, some recently developed methods are special cases of our result.

\section{DEVELOPMENT OF METHODS CONVERGENCE ANALYSIS}

Throughout this paper, we consider iterative methods to find a simple root $\beta$, i.e., $f(\beta)=0$ and $f^{\prime}(\beta) \neq 0$ of a nonlinear equation $f(x)=0$.

*Address correspondence to this author at the Institute of Operational Research \& Cybernetics Hangzhou Dianzi University, Hangzhou, 310018, P.R. China; Tel: +8657181792565 ;

Fax: 86571869190030; E-mail: weili@hdu.edu.cn
Let $\phi_{p}\left(x_{n}\right)$ be any iteration function, whose order of convergence is at least $p$, which means that the corresponding iterarive method defined by

$z_{n}=\phi_{p}\left(x_{n}\right), n=0,1,2 \cdots$

is of order $p$ and satisfies the error equation

$z_{n}-\beta=A e_{n}^{p}+O\left(e_{n}^{p+1}\right)$.

where $e_{n}=x_{n}-\beta$ and $A$ is constant.

Now we suggest and analyze the following iterative method for solving nonlinear equation:

$\left\{\begin{array}{l}y_{n}=x_{n}-\frac{f\left(x_{n}\right)}{f^{\prime}\left(x_{n}\right)}, \\ z_{n}=\phi_{p}\left(x_{n}\right), \\ x_{n+1}=z_{n}-H(T) \frac{f\left(z_{n}\right)}{f^{\prime}\left(x_{n}\right)}, n=0,1,2 \cdots,\end{array}\right.$

where $T=\frac{f^{\prime}\left(y_{n}\right)}{f^{\prime}\left(x_{n}\right)}$ and $H(t)$ denotes a single-variable function to be determined later. Consider the conditions below.

$H(1)=1$,

$H^{\prime}(1)=-1$,

$H^{\prime \prime}(1)=3$.

Our concern here is to find $H(t)$ for which the method defined by (3) has higher order of convergence. This can be answered in the following theorem, which gives a detailed analysis of convergence. The method is similar to the method presented in [36] in which it introduces the auxiliary function $H$. However, instead of using the two-variable function $H(x, y)$ in [36], a much more simpler single variable function $H(t)$ is used in the algorithm. This simplification is owing to the usage of a novel ratio $T=\frac{f^{\prime}\left(y_{n}\right)}{f^{\prime}\left(x_{n}\right)}$. 


\section{Theorem 1}

Let $\beta$ be a simple zero of sufficient differentiable function $f$. If $H(t)$ satisfies condition (4) and $\left|H^{\prime}(1)\right|<\infty$, then the iterative method defined by (3) is of order at least $p+1$ and satisfies the error equation

$e_{n+1}=A\left(\left(2 c_{2}+2 H^{\prime}(1) c_{2}\right) e_{n}^{P+1}\right)+0\left(e_{n}^{P+2}\right)$.

If $H(t)$ satisfies conditions (4), (5) and $\left|H^{\prime \prime}(1)\right|<\infty$, then the iterative method defined by (3) is of order at least $p+2$ and satisfies the error equation

$e_{n+1}=A\left(6 c_{2}^{2}-2 H^{\prime \prime}(1) c_{2}^{2}\right) e_{n}^{P+2}+0\left(e_{n}^{P+3}\right)$.

If $H(t)$ satisfies condition (4-6) and $\left|H^{(3)}(t)\right|<\infty$, then the iterative method defined by (3) is of order at least $\min \{2 p, p+3\}$ and satisfies the error equation

$e_{n+1}=A\left(20 c_{2}^{3}-2 c_{2} c_{3}+\frac{4}{3} c_{2}^{3} H^{(3)}(1)\right) e_{n}^{P+3}-$

$c_{2} A^{2} e_{n}^{2 p}+0\left(e_{n}^{P+4}\right)$.

where $c_{k}=\frac{f^{(k)}(\beta)}{f^{\prime}(\beta) k !}, \quad(k=2,3 \cdots)$.

Proof. Let

$z_{n}-\beta=A e_{n}^{P}+0\left(e_{n}^{P+1}\right)$

Using Taylor series expansion of $f\left(z_{n}\right)$ about the simple zero $\beta$ and taking into account (10), we have

$$
\begin{aligned}
f\left(z_{n}\right) & \left.=\begin{array}{l}
f^{\prime}(\beta)\left(z_{n}-\beta\right)\left[1+c_{2}\left(z_{n}-\beta\right)+\right. \\
\end{array}\left(\left(z_{n}-\beta\right)^{2}\right)\right] \\
& =f^{\prime}(\beta)\left(z_{n}-\beta\right)\left[1+c_{2} A e_{n}^{p}+0\left(e_{n}^{p+1}\right)\right]
\end{aligned}
$$

By the Taylor expansion of $f\left(x_{n}\right)$ and $f^{\prime}\left(x_{n}\right)$ about $\beta$ respectively, we get

$$
\begin{aligned}
& f\left(x_{n}\right)=f^{\prime}(\beta)\left(e_{n}+c_{2} e_{n}^{2}+c_{3} e_{n}^{3}+0\left(e_{n}^{4}\right),\right. \\
& f^{\prime}\left(x_{n}\right)=f^{\prime}(\beta)\left(1+2 c_{2} e_{n}+3 c_{3} e_{n}^{2}+4 c_{4} e_{n}^{3}+0\left(e_{n}^{4}\right)\right) .
\end{aligned}
$$

Then, dividing (12) by (13) gives

$$
\frac{f\left(x_{n}\right)}{f^{\prime}\left(x_{n}\right)}=e_{n}-c_{2} e_{n}^{2}+2\left(c_{2}^{2}-c_{3}\right) e_{n}^{3}+c_{2} A e_{n}^{p}+o\left(e_{n}^{4}\right),
$$

and hence

$$
\begin{aligned}
& y_{n}=\beta+c_{2} e_{n}^{2}-2\left(c_{2}^{2}-c_{3}\right) e_{n}^{3} \\
& -\left(7 c_{2} c_{3}-4 c_{2}^{3}-3 c_{4}\right) e_{n}^{4}+o\left(e_{n}^{5}\right), \\
& \frac{f\left(z_{n}\right)}{f^{\prime}\left(x_{n}\right)}=\left(z_{n}-\beta\right)\left[1-2 c_{2} e_{n}+\left(4 c_{2}^{2}-3 c_{3}\right) e_{n}^{2}+\right. \\
& \left.\left(c_{2} A+12 c_{2} c_{3}-8 c_{2}^{3}-4 c_{4}\right) e_{n}^{3}+0\left(e_{n}^{4}\right)\right] .
\end{aligned}
$$

Note that

$$
T=\frac{f^{\prime}\left(y_{n}\right)}{f^{\prime}\left(x_{n}\right)}=1-2 c_{2} e_{n}+\left(6 c_{2}^{2}-3 c_{3}\right) e_{n}^{2}+
$$$$
\left(16 c_{2} c_{3}-16 c_{2}^{3}-4 c_{4}\right) e_{n}^{3}+0\left(e_{n}^{4}\right),
$$

we obtain

$$
\begin{aligned}
H(T)= & H(1)+H^{\prime}(1)(T-1)+\frac{H^{\prime \prime}(1)}{2}(T-1)^{2} \\
& +\frac{H^{(3)}(1)}{6}(T-1)^{3}+0\left((T-1)^{4}\right) \\
= & H(1)-2 H^{\prime}(1) c_{2} e_{n}+\left[H^{\prime}(1)\left(6 c_{2}^{2}-2 c_{3}\right)\right. \\
& \left.+2 H^{\prime \prime}(1) c_{2}^{2}\right] e_{n}^{2} \\
& +\left[H^{\prime}(1)\left(16 c_{2} c_{3}-16 c_{2}^{3}-4 c_{4}\right)-2 H^{\prime \prime}\right. \\
& \left.(1) c_{2}\left(6 c_{2}^{2}-3 c_{3}\right)-\frac{4}{3} H^{(3)}(1) c_{2}^{2}\right] e_{n}^{3}+0\left(e_{n}^{4}\right) .
\end{aligned}
$$

Thus, from (16) and (18), we obtain

$$
\begin{aligned}
H(T) \frac{f\left(z_{n}\right)}{f^{\prime}\left(x_{n}\right)}= & \left(z_{n}-\beta\right)\left[H(1)-\left(2 H(1) c_{2}\right.\right. \\
& \left.+2 H^{\prime}(1) c_{2}\right) e_{n} \\
& +\left[H^{\prime}(1)\left(10 c_{2}^{2}-3 c_{3}\right)+2 H^{\prime \prime}\right. \\
& \left.(1) c_{2}^{2}+H(1)\left(4 c_{2}^{2}-3 c_{3}\right)\right] e_{n}^{2} \\
& +\left[H^{\prime}(1)\left(28 c_{2} c_{3}-36 c_{2}^{3}-4 c_{4}\right)\right. \\
& +H^{\prime \prime}(1)\left(6 c_{2} c_{3}-16 c_{2}^{3}\right) \\
& +H(1)\left(12 c_{2} c_{3}-8 c_{2}^{3}-4 c_{4}\right)-\frac{4}{3} H^{(3)} \\
& \left.\left.(1) c_{2}^{2}\right] e_{n}^{3}+H(1) c_{2} A e_{n}^{p}+0\left(e_{n}^{4}\right)\right] .
\end{aligned}
$$

If $H(t)$ satisfies the condition (4), we have

$$
\begin{aligned}
& z_{n}-H(T) \frac{f\left(z_{n}\right)}{f^{\prime}\left(x_{n}\right)}=\beta+A\left(2 c_{2}+2 H^{\prime}(1) c_{2}\right) \\
& e_{n}^{p+1}+O\left(e_{n}^{p+2}\right),
\end{aligned}
$$

and hence

$$
e_{n+1}=A\left(2 c_{2}+2 H^{\prime}(1) c_{2}\right) e_{n}^{p+1}+O\left(e_{n}^{p+2}\right) \text {. }
$$

If $H(t)$ satisfies the conditions (4) and (5), we have

$z_{n}-H(T) \frac{f\left(z_{n}\right)}{f^{\prime}\left(x_{n}\right)}=\beta+A\left(6 c_{2}^{2}-2 H^{\prime \prime}(1) c_{2}^{2}\right)$

$e_{n}^{p+2}+0\left(e_{n}^{p+3}\right)$,

and hence

$e_{n+1}=A\left(6 c_{2}^{2}-2 H^{\prime \prime}(1) c_{2}^{2}\right) e_{n}^{p+2}+0\left(e_{n}^{p+3}\right)$.

If $H(t)$ satisfies the conditions (4)-(6), we have

$$
\begin{aligned}
H(T) \frac{f\left(z_{n}\right)}{f^{\prime}\left(x_{n}\right)}= & \begin{array}{l}
\left(z_{n}-\beta\right)\left[1+\left(2 c_{2} c_{3}-20 c_{2}^{3}-\frac{4}{3} H^{(3)}\right.\right. \\
\left.\left.(1) c_{2}^{2}\right) e_{n}^{3}+c_{2} A e_{n}^{p}+0\left(e_{n}^{p+1}\right)\right]
\end{array} \\
= & z_{n}-\beta+A\left(2 c_{2} c_{3}-20 c_{2}^{3}-\frac{4}{3} H^{(3)}\right. \\
& \left.(1) c_{2}^{2}\right) e_{n}^{(p+3)}+c_{2} A^{2} e_{n}^{2 p}+0\left(e_{n}^{p+4}\right) .
\end{aligned}
$$


$z_{n}-H(T) \frac{f\left(z_{n}\right)}{f^{\prime}\left(x_{n}\right)}=\beta+A\left(20 c_{2}^{3}-2 c_{2} c_{3}+\right.$

$\left.\frac{4}{3} H^{(3)}(1) c_{2}^{3}\right) e_{n}^{p+3}-c_{2} A^{2} e_{n}^{2 p}+0\left(e_{n}^{p+4}\right)$,

Thus,

$e_{n+1}=A\left(20 c_{2}^{3}-2 c_{2} c_{3}+\frac{4}{3} c_{2}^{3} H^{(3)}(1)\right) e_{n}^{p+3}$.

$-c_{2} A^{2} e_{n}^{2 p}+0\left(e_{n}^{p+4}\right)$,

this completes the proof.

As it can be seen from the Theorem, that the order of any existing iterative method with order $p$ can be improved to $p+1, p+2$ or $\min \{2 p, p+3\}$ more higher, respectively. The expense of this acceleration based on only one additional function evaluation or two additional function evaluations, depends on that the iteration formula $z_{n}=\phi_{p}\left(x_{n}\right)$ either including the function evaluation $f^{\prime}\left(y_{n}\right)$ or not.

The Theorem provides us with a unified frame to construct new higher-order methods, which improve the computational efficiency of the original method much better.

\section{SOME ITERATIVE METHODS DEVELOPED BY THEOREM 1}

In this section, we show some recently developed methods which are special cases of the method (3). Also, many new iterative methods can be developed by choosing different function $H(t)$.

\subsection{Relations with Some Recently Developed Iterative Methods}

Let

$H(t)=\frac{t+1}{3 t-1}$.

It is easy to show that the function $H(t)$ satisfies the conditions (4)-(6). If we take the iteration function,

$z_{n}=\phi_{3}\left(x_{n}\right)=x_{n}-\frac{2 f\left(x_{n}\right)}{f^{\prime}\left(x_{n}\right)+f^{\prime}\left(y_{n}\right)}$,

which is the known iterative function of order three [16], then by Theorem 1 we obtain the following iterative method,

$$
\left\{\begin{array}{l}
y_{n}=x_{n}-\frac{f\left(x_{n}\right)}{f^{\prime}\left(x_{n}\right)} \\
z_{n}=x_{n}-\frac{2 f\left(x_{n}\right)}{f^{\prime}\left(x_{n}\right)+f^{\prime}\left(y_{n}\right)} \\
x_{n+1}=z_{n}-\frac{f\left(z_{n}\right)}{f^{\prime}\left(x_{n}\right)} \frac{f^{\prime}\left(x_{n}\right)+f^{\prime}\left(y_{n}\right)}{3 f^{\prime}\left(y_{n}\right)-f^{\prime}\left(x_{n}\right)}, n=0,1,2 \cdots
\end{array}\right.
$$

which converges with order 6 . This is the main result given by S.K. Parhi and D.K. Gupta [35].
If we take the iteration function,

$$
z_{n}=\phi_{3}\left(x_{n}\right)=x_{n}-\frac{f\left(x_{n}\right)}{2}\left(\frac{1}{f^{\prime}\left(x_{n}\right)}+\frac{1}{f^{\prime}\left(y_{n}\right)}\right),
$$

which is the iterative function of order three [21, 23], then by Theorem 1 we obtain the following sixth-order iterative method

$$
\left\{\begin{array}{l}
y_{n}=x_{n}-\frac{f\left(x_{n}\right)}{f^{\prime}\left(x_{n}\right)} \\
z_{n}=x_{n}-\frac{f\left(x_{n}\right)}{2}\left(\frac{1}{f^{\prime}\left(x_{n}\right)}+\frac{1}{f^{\prime}\left(y_{n}\right)}\right) \\
x_{n+1}=z_{n}-\frac{f\left(z_{n}\right)}{f^{\prime}\left(x_{n}\right)} \frac{f^{\prime}\left(x_{n}\right)+f^{\prime}\left(y_{n}\right)}{3 f^{\prime}\left(y_{n}\right)-f^{\prime}\left(x_{n}\right)}, n=0,1,2 \cdots .
\end{array}\right.
$$

This is the main result given by Jisheng kou[27].

If we choose

$H(t)=\frac{2 t}{t^{2}+2 t-1}$

and the iterative functions (27) and (29), by applying the theorem we obtain

$$
\left\{\begin{array}{l}
y_{n}=x_{n}-\frac{f\left(x_{n}\right)}{f^{\prime}\left(x_{n}\right)} \\
z_{n}=x_{n}-\frac{2 f\left(x_{n}\right)}{f^{\prime}\left(x_{n}\right)+f^{\prime}\left(y_{n}\right)} \\
x_{n+1}=z_{n}-\frac{2 f^{\prime}\left(y_{n}\right) f\left(z_{n}\right)}{f^{\prime}\left(y_{n}\right)^{2}+2 f^{\prime}\left(y_{n}\right) f^{\prime}\left(x_{n}\right)-f^{\prime}\left(x_{n}\right)^{2}}, \\
n=0,1,2 \cdots
\end{array}\right.
$$

and

$$
\left\{\begin{array}{l}
y_{n}=x_{n}-\frac{f\left(x_{n}\right)}{f^{\prime}\left(x_{n}\right)} \\
z_{n}=x_{n}-\frac{f\left(x_{n}\right)}{2}\left(\frac{1}{f^{\prime}\left(x_{n}\right)}+\frac{1}{f^{\prime}\left(y_{n}\right)}\right) \\
x_{n+1}=z_{n}-\frac{2 f^{\prime}\left(y_{n}\right) f\left(z_{n}\right)}{f^{\prime}\left(y_{n}\right)^{2}+2 f^{\prime}\left(y_{n}\right) f^{\prime}\left(x_{n}\right)-f^{\prime}\left(x_{n}\right)^{2}}, \\
n=0,1,2 \cdots,
\end{array}\right.
$$

separately. These are also new methods developed recently by Jisheng kou [27].

\subsection{Some New Iterative Methods}

It is easy to find different $H(t)$ satisfying condition (4)-(6), this can be done with the help of mathematical packages, such as Maple, Mathematica. Thus, many existed iterative methods belong to this unified framework. Also, many new higher-order methods can be developed by using Theorem 1. For example, we may find function 
$H(t)=\frac{t^{2}-2 t+3}{2 t}, H(t)=\frac{3 t^{2}-8 t+7}{2}$, etc, satisfying condition

(4)-(6). Now consider the function;

$H(t)=\frac{t^{2}-2 t+3}{2 t}$

and combine it with the iterative functions

$$
z_{n}=\phi_{3}\left(x_{n}\right)=x_{n}-\frac{2 f\left(x_{n}\right)}{f^{\prime}\left(x_{n}\right)+f^{\prime}\left(y_{n}\right)}
$$

and

$z_{n}=\phi_{3}\left(x_{n}\right)=x_{n}-\frac{f\left(x_{n}\right)}{2}\left(\frac{1}{f^{\prime}\left(x_{n}\right)}+\frac{1}{f^{\prime}\left(y_{n}\right)}\right)$

respectively. Thus, we obtain two new sixth-order methods,

$$
\left\{\begin{array}{l}
y_{n}=x_{n}-\frac{f\left(x_{n}\right)}{f^{\prime}\left(x_{n}\right)} \\
z_{n}=x_{n}-\frac{2 f\left(x_{n}\right)}{f^{\prime}\left(x_{n}\right)+f^{\prime}\left(y_{n}\right)} \\
x_{n+1}=z_{n}-\frac{f^{\prime}\left(y_{n}\right)^{2}-2 f^{\prime}\left(y_{n}\right) f^{\prime}\left(x_{n}\right)+3 f^{\prime}\left(x_{n}\right)^{2}}{2 f^{\prime}\left(y_{n}\right) f^{\prime}\left(x_{n}\right)} \frac{f\left(z_{n}\right)}{f^{\prime}\left(x_{n}\right)} \\
n=0,1,2 \cdots
\end{array},\right.
$$

and

$$
\left\{\begin{array}{l}
y_{n}=x_{n}-\frac{f\left(x_{n}\right)}{f^{\prime}\left(x_{n}\right)} \\
z_{n}=x_{n}-\frac{f\left(x_{n}\right)}{2}\left(\frac{1}{f^{\prime}\left(x_{n}\right)}+\frac{1}{f^{\prime}\left(y_{n}\right)}\right) \\
x_{n+1}=z_{n}-\frac{f^{\prime}\left(y_{n}\right)^{2}-2 f^{\prime}\left(y_{n}\right) f^{\prime}\left(x_{n}\right)+3 f^{\prime}\left(x_{n}\right)^{2}}{2 f^{\prime}\left(y_{n}\right) f^{\prime}\left(x_{n}\right)} \frac{f\left(z_{n}\right)}{f^{\prime}\left(x_{n}\right)} \\
n=0,1,2 \cdots
\end{array}\right.
$$

respectively.

Similarly, consider the function $H(t)=\frac{-2}{1-4 t+t^{2}}$ and the equation (34), we obtain another new sixth-order method

$$
\left\{\begin{array}{l}
y_{n}=x_{n}-\frac{f\left(x_{n}\right)}{f^{\prime}\left(x_{n}\right)} \\
z_{n}=x_{n}-\frac{f\left(x_{n}\right)}{2}\left(\frac{1}{f^{\prime}\left(x_{n}\right)}+\frac{1}{f^{\prime}\left(y_{n}\right)}\right) \\
x_{n+1}=z_{n}+\frac{2 f^{\prime}\left(x_{n}\right) f\left(z_{n}\right)}{f^{\prime}\left(x_{n}\right)^{2}-4 f^{\prime}\left(y_{n}\right) f^{\prime}\left(x_{n}\right)+f^{\prime}\left(y_{n}\right)^{2}}, \\
n=0,1,2 \ldots
\end{array}\right.
$$

These new sixth-order methods add only one evaluation of the function at the point iterated by the third-order methods to obtain the sixth-order, so that they have the efficiency index equal to $\sqrt[4]{6} \approx 1.565$, which is much better than that of Newton's method $\sqrt{2} \approx 1.414$. One may try to find some other new iterative methods by using the methodology described in section 2 .

\section{NUMERICAL EXAMPLES}

To illustrate the convergence speed and behavior of some methods from the proposed families ((3)), we have tested a number of algebraic polynomials and analytic functions. For all test problems the stop criteria is $\left|f\left(x_{n+1}\right)\right|<10^{-14}$.

The Newton method (NM), Neta's 6th order method [3] (Neta), Jisheng Kou et al's method [28] (Kou), the method defined by (27) (AM), (28) (AM1), (29) (HM), (32) (HM1) and the new method obtained in this paper (35) (AM2), (36) (HM2) and (37) (HM3). We used the following test functions and display the approximate zeros $\beta$ found up to the 15 th decimal place.

$$
\begin{aligned}
& f_{(1)}(x)=x^{3}+4 x^{2}-10, \beta=1.365230013414097 \\
& f_{(2)}(x)=\sin x^{2}-x^{2}+1, \beta=1.404491648215341 \\
& f_{(3)}(x)=x e^{x^{2}}-\sin x^{2}+3 \cos x+5, \beta=-1.20764- \\
& 7827130919 \\
& f_{(4)}(x)=(x-1)^{3}-1, \beta=2 \\
& f_{(5)}(x)=e^{x^{2}+7 x-30}-1, \beta=3 \\
& f_{(6)}(x)=x^{3}-10, \beta=2.154434690031884 \\
& f_{(7)}(x)=x^{2}-e^{x}-3 x+2, \beta=0.257530285439861 \\
& f_{(8)}(x)=(x-2)^{23}-1, \beta=3
\end{aligned}
$$

The results of the comparison of the number of iteration of various iterative methods and Newton's method. $x_{0}$ is the given initial point are displayed in Table $\mathbf{1}$.

The test results in Table $\mathbf{1}$ show that the computational orders of convergence of the newly proposed methods are in accordance with the theory developed in the Section 2. For most of the functions we tested, the methods introduced in the present presentation behave at least equal performance as compared to the other well known methods of the same order of convergence, and can compete with Newton's method also.

Neta's sixth order method [3] performs well with these test problems. The three new sixth order methods are as good as Neta's 6th order method for first 7 test problems, except for the eighth test equation. It should be pointed out that these three new methods are only arbitrarily chosen illustrative examples for using the proposed framework. Thus, we do not want to claim too much about the superiority of these three new methods based on our computational tests. However, some very efficient methods might be developed by using the proposed framework. In fact, it is interesting to see that for these test functions, the algorithm AM1, proposed by S.K. Parhi and D.K. Gupta [35], is the best. Note that AM1 is a special case of our 
Table 1. Comparison of the Number of Iteration

\begin{tabular}{|c|c|c|c|c|c|c|c|c|c|c|c|}
\hline \multirow[t]{2}{*}{ FUC } & \multirow[t]{2}{*}{$x_{0}$} & \multicolumn{10}{|c|}{ Number of Iteration } \\
\hline & & NM & Neta & Kou & $\mathbf{A M}$ & AM1 & AM2 & HM & HM1 & HM2 & HM3 \\
\hline \multirow{2}{*}{$f_{(1)}(x)$} & 1 & 5 & 2 & 2 & 3 & 2 & 2 & 3 & 2 & 2 & 2 \\
\hline & 2 & 5 & 2 & 2 & 3 & 2 & 2 & 3 & 2 & 2 & 2 \\
\hline \multirow{2}{*}{$f_{(2)}(x)$} & 1 & 6 & 2 & 2 & 4 & 2 & 3 & 3 & 2 & 3 & 3 \\
\hline & 3 & 6 & 3 & 3 & 3 & 2 & 2 & 2 & 3 & 3 & 2 \\
\hline \multirow{2}{*}{$\begin{array}{l}f_{(3)}(x) \\
f_{(4)}(x)\end{array}$} & -2 & 8 & 4 & 4 & 6 & 4 & 4 & 5 & DIV & 4 & 3 \\
\hline & 2.5 & 6 & 2 & 2 & 4 & 2 & 3 & 3 & 2 & 2 & 3 \\
\hline \multirow[b]{2}{*}{$f_{(5)}(x)$} & 3.5 & 7 & 3 & 3 & 5 & 3 & 3 & 4 & 3 & 3 & 3 \\
\hline & 3.25 & 8 & 3 & 4 & 6 & 3 & 4 & 5 & DIV & 4 & 3 \\
\hline \multirow[b]{2}{*}{$f_{(6)}(x)$} & 3.5 & 12 & 5 & 5 & 8 & 4 & 5 & 7 & DIV & 5 & 5 \\
\hline & 1.5 & 6 & 2 & 2 & 4 & 2 & 3 & 3 & 2 & 3 & 3 \\
\hline \multirow{2}{*}{$f_{(7)}(x)$} & 2 & 5 & 3 & 4 & 4 & 3 & 3 & 4 & 3 & 3 & 3 \\
\hline & 3 & 5 & 4 & 3 & 4 & 3 & 3 & 3 & 3 & 3 & 4 \\
\hline \multirow[t]{2}{*}{$f_{(8)}(x)$} & 3.5 & 14 & 4 & 6 & 10 & 4 & 6 & 8 & DIV & DIV & 6 \\
\hline & 4.5 & 26 & 7 & 15 & 17 & 7 & 12 & 14 & DIV & 11 & 10 \\
\hline
\end{tabular}

approach. Therefore, it is valuable to do further study to construct efficient algorithms under the proposed framework.

\section{CONCLUSIONS}

In this work we develop a unified framework to construct higher-order iterative methods for solving nonlinear equations. The proposed methods add only one or two function evaluations at the point iterated by the existing p-order iteration formula but they increase the order of the existing method to $\min \{2 p, p+3\}$ units higher.

By considering many other possible combinations of the p-order formulas and the functions $H$ satisfying condition (4-6), we can continuously derive many new high-order methods.

An interesting question is that, what would happen if higher order derivatives of $H(t)$ would be considered?

Would it not be possible to compute the order of the method if higher derivatives of $H(t)$ and their bounds would be considered? From the proof of the Theorem 1, it is clear that (4-6) should be used if we want to increase the order of the existing method to $\min \{2 p, p+3\}$ units higher based on the present framework. Also, the result can not be improved even if the higher derivatives of $H(t)$ and their bounds are considered. In fact, the coefficients of $e_{n}^{p+3}$ and $e_{n}^{2 p}$ in the equation (26) can not be vanished by choosing the values of higher derivatives of $H(t)$. Thus, the new framework is required if one want to exploit the higher higher derivatives of $H(t)$, that will be an interesting fields for future studies.

\section{ACKNOWLEDGEMENTS}

This work was partially supported by NSF of China (Mathematic Tian Yuan fund) 10926058.

The authors wish to thank the referees for some very crucial comments and suggestions that helped us to improve the quality of the paper.

We are taking the liberty of presenting the comment written by one of the referees. He wrote:

The reviewer, however, likes to add some comments from an engineering point of view. From this perspective the reduced number of iteration steps may be a nice feature but it is not essential for the presented examples. However, the proposed method could be a valuable contribution, if the proposed algorithm can be applied to the solution of a system of nonlinear equations, which repeatedly needs to be solved at every integration point of a FE-mesh, when nonlinear constitutive behaviour is taken into account. In this context it is extremely important that (i) no divergence occurs (as is the case for algorithm HM1 even for a nonlinear scalar equation) and that (ii) the algorithm is very efficient. The latter property is not only reflected by the number of iteration steps but also by the number of operations and computing time. E.g., if one iteration step 
requires much more computing time then the smaller number of iteration steps is overruled.

\section{REFERENCES}

[1] Ostrowski AM. Solution of equations and systems of equations. New York: Academic Press 1960.

[2] King R. A family of fourth-order methods for nonlinear equations. SIAM J Numer Anal 1973; 10: 876-9.

[3] Neta B. A sixth-order family of methods for nonlinear equations. Int J Comput Math 1979; 7: 157-61.

[4] Popovski DB. A hybrid algorithm for finding roots. Informatica 1979; 3: 16-17.

[5] Popovski DB. A root finding algorithm. Prilozi 1979; 30-31: 28993.

[6] Popovski DB. On an algorithm for finding function zeros (SerboCroation). Informatica1980; 1: 47-8.

[7] Popovski DB. A Note on Neta's Family of sixth-order methods for solving equations. International J Comput Math 1981; 10: 913.

[8] Popovski DB. Sixth order methods for solving equations. J Appl Math Phys 1982; 33: 434-8.

[9] Igarashi M. A termination criterion for iteration methods used to find the zeros of polynomial. Math Comp 1984; 42: 165-71.

[10] Gander W. On Halley's iteration method. Am Math Monthly 1985; 90: 131-4.

[11] Argyros LK. A note on the Halley method in Banach spaces. Appl Math Comput 1993; 58: 215-24.

[12] Argyros LK, Chen D, Qian Q. The Jarratt method in Banach space setting. J Appl Math Comput 1994; 51: 103-6.

[13] Ford WF, Pennline JA. Accelerated convergence in Newton's method. SIAM Rev 1996; 38: 658-9.

[14] Gutieérrez JM, Hernaández MA. A family of Chebyshev-Halley type methods in Banach spaces. Bull Aust Math Soc 1997; 55: 11330.

[15] He GH. Newton-like iteration methods for solving algebraic equations. Commun Nonlinear Sci Numer Simul 1998; 3: 106-9.

[16] Weerakoon S, Fernando TGI. A variant of Newton's method with accelerated third-order convergence. Appl Math Lett 2000; 13: 8793.

[17] Wu XY. A new continuation of Newton-like method and its deformation. Appl Math Comput 2000; 112: 75-8.

[18] Gutieérrez JM. Hernaández MA. An acceleration of Newtons method:super-Halley method. Appl Math Comput 2001; 117: 22339.
[19] Abbasbandy S. Improving Newton-Raphson method for nonlinear equations by modified Adomian decomposition method. Appl Math Comput 2003; 145: 887-93.

[20] Frontini M, Sormani E. Modifed Newton's method with third-order convergence and multiple roots. J Comput Appl Math 2003; 156: 345-54.

[21] Özban AY. Some new variants of Newton's method. Appl Math Lett 2004; 17: 677-82.

[22] Chun C. Iterative methods improving Newton's method by the decomposition method. Comput Math Appl 2005; 50: 1559-68.

[23] Homeier HHH. On Newton-type methods with cubic convergence. J Comput Appl Math 2005; 176: 425-32.

[24] Chen J. Some new iterative methods with three-order convergence. Appl Math Lett 2006; 181: 1519-22.

[25] Basto M, Semiao V, Calheiros FL. A new iterative method to compute nonlinear equations. Appl Math Comput 2006; 173: 46883 .

[26] Aslam NM, Inayat NK. Three-step iterative methods for nonlinear equations. Appl Math Comput 2006; 183: 322-7.

[27] Kou J. The improvements of modified Newton's method. Appl Math Comput 2007; 189: 602-9.

[28] Kou J, Li Y. An improvement of Jarratt method. Appl Math Comput 2007; 189: 1816-21.

[29] Kou J, Li Y, Wang X. Some modifications of Newton's method with fifth-order convergence. J Comput Appl Math 2007; 209: 14652.

[30] Petković LD, Petković MS. A note on some recent methods for solving nonlinear equations. Appl Math Comput 2007; 185: 368-74.

[31] Chakravarthy PP, Phaneendra K, Reddy YN. A seventh order numerical method for singular perturbation problems. Appl Math Comput 2007; 186: 860-71.

[32] Basu D. From third to fourth order variant of Newton's method for simple roots. Appl Math Comput 2008; 202: 886-92.

[33] Yun JH. A note on three-step iterative method for nonlinear equations. Appl Math Comput 2008; 202: 401-5.

[34] Hueso JL, Martínez E, Torregrosa JR. A note on some three-step iterative methods for nonlinear equations. Appl Math Comput 2008; 202: 252-5

[35] Parhi SK, Gupta DK. A sixth order method for nonlinear equations. Appl Math Comput 2008; 203: 50-5.

[36] Ham YM, Chun C, Lee S-G. Some higher-order modifications of Newton's method for solving nonlinear equations. J Comput Appl Math 2008; 222: 477-86.

[37] Maheshwar AK. A fourth order iterative method for solving nonlinear equations. Appl Math Comput 2009; 211: 383-91. 